\title{
SOME GENERATING-FUNCTION EQUIVALENCES $\dagger$
}

\author{
by H. M. SRIVASTAVA
}

(Received 15 April, 1974)

A generalization is given of a theorem of F. Brafman [1] on the equivalence of generating relations for a certain sequence of functions. The main result, contained in Theorem 2 below, may be applied to several special functions including the classical orthogonal polynomials such as Hermite, Jacobi (and, of course, Legendre and ultraspherical), and Laguerre polynomials.

1. Let a sequence of functions $f_{n}(x), n=0,1,2, \ldots$, be defined by the Rodrigues formula

$$
f_{n}(x)=\frac{1}{n !} D_{x}^{n}\left\{(a x+b)^{n} F(x)\right\}, D_{x}=d / d x,
$$

where $a$ and $b$ are constants, not both zero, and $F(x)$ is independent of $n$ and differentiable an arbitrary number of times. The following result is due to F. Brafman [1].

THEOREM 1. If a generating function

$$
\sum_{n=0}^{\infty} a_{n} f_{n}(x) t^{n}
$$

is known for either

$$
a_{n}={ }_{p+1} F_{q}\left[\begin{array}{c}
-n, \alpha_{1}, \ldots, \alpha_{p} ; \\
\beta_{1}, \ldots, \beta_{q} ;
\end{array}\right]
$$

or

$$
a_{n}=\frac{\left(\alpha_{1}\right)_{n} \ldots\left(\alpha_{p}\right)_{n}}{\left(\beta_{1}\right)_{n} \ldots\left(\beta_{q}\right)_{n}}
$$

then it is automatically known for the other.

Also a further result holds connecting a generating function of the set $f_{n}(x)$ with one of the $\operatorname{set}_{2 n}(x)$.

Brafman's proof of Theorem 1 involves contour integration and makes use of Cauchy's integral formula and two known generating relations for certain hypergeometric polynomials (cf. [1], pp. 156-158). It may be of interest to observe that a substantially more general generating-function equivalence than what is contained in Theorem 1 would follow fairly easily from Lagrange's theorem [3, p. 133]

tSupported in part by NRC grant A-7353. 


$$
\frac{f(z)}{1-t \phi^{\prime}(z)}=\sum_{n=0}^{\infty} \frac{t^{n}}{n !} D_{x}^{n}\left\{[\phi(x)]^{n} f(x)\right\}, z=x+t \phi(z) .
$$

Indeed we first obtain the following

LEMMA. For every sequence $\left\{f_{n}(x)\right\}$ defined by (1), the generating relation

$$
\sum_{n=0}^{\infty}\left(\begin{array}{c}
m+n \\
n
\end{array}\right) f_{m+n}(x) t^{n}=(1-a t)^{-m-1} f_{m}\left(\frac{x+b t}{1-a t}\right)
$$

holds when $m=0,1,2, \ldots$.

To prove this lemma we notice from (1) and (5) that

$$
\begin{aligned}
\sum_{n=0}^{\infty}\left(\begin{array}{c}
m+n \\
n
\end{array}\right) f_{m+n}(x) t^{n} & =\frac{1}{m !} D_{x}^{m} \sum_{n=0}^{\infty} \frac{t^{n}}{n !} D_{x}^{n}\left\{(a x+b)^{m+n} F(x)\right\} \\
& =\frac{(1-a t)^{-1}}{m !} D_{x}^{m}\left\{(a z+b)^{m} F(z)\right\} \\
& =\frac{(1-a t)^{-m-1}}{m !} D_{z}^{m}\left\{(a z+b)^{m} F(z)\right\},
\end{aligned}
$$

since $z=(x+b t) /(1-a t)$, and the generating relation (6) follows by appealing to the definition (1) once again.

We now state our main result given by

THEOREM 2. For arbitrary coefficients $\lambda_{n}, n=0,1,2, \ldots$, and integer $N \geqq 1$, if we let a generating function

$$
\sum_{n=0}^{\infty} A_{n} f_{r n}(x) t^{n}
$$

be known for either

$$
A_{n}=\sum_{k=0}^{[n / N]}\left(\begin{array}{c}
n \\
N k
\end{array}\right) \lambda_{k} y^{k} \text { and } \quad r=1
$$

or

$$
A_{n}=\lambda_{n} \text { and } r=N
$$

then it is automatically known for the other.

Proof. From (7) and (8) we have

$$
\begin{aligned}
\Omega \equiv \sum_{n=0}^{\infty} A_{n} f_{n}(x) t^{n} & =\sum_{n=0}^{\infty} f_{n}(x) t^{n} \sum_{k=0}^{[n / N]}\left(\begin{array}{c}
n \\
N k
\end{array}\right) \lambda_{k} y^{k} \\
& =\sum_{k=0}^{\infty} \lambda_{k}\left(y t^{N}\right)^{k} \sum_{n=0}^{\infty}\left(\begin{array}{c}
n+N k \\
N k
\end{array}\right) f_{n+N k}(x) t^{n}
\end{aligned}
$$


by inverting the order of summations. This process may be justified when the series involved converge absolutely. However, the series need not converge, and in such cases a divergent generating function is formally obtained.

Now apply the lemma with $m=N k$, where $N-1, k=0,1,2, \ldots$, in order to sum the inner series, and we find that

$$
\Omega=(1-a t)^{-1} \sum_{k=0}^{\infty} \lambda_{k} f_{N k}\left(\frac{x+b t}{1-a t}\right)\left[\frac{y t^{N}}{(1-a t)^{N}}\right]^{k},
$$

whose second member involves a generating function of the type given by (7) and (9).

This evidently completes the proof of Theorem 2 , which provides an elegant connection between a generating function of the set $f_{n}(x)$ with one of the set $f_{N n}(x)$, where $N$ is an arbitrary positive integer.

Alternatively, to prove Theorem 2, the principle illustrated in [1] may be applied mutatis mutandis. Indeed, if $C$ denotes a simple closed contour about $z=x$, then from (1) and Cauchy's integral formula we readily have

$$
f_{n}(x)=\frac{1}{2 \pi i} \int_{C} \frac{(a z+b)^{n} F(z)}{(z-x)^{n+1}} d z, \quad n=0,1,2, \ldots,
$$

whence

$$
\begin{aligned}
\Omega & =\sum_{n=0}^{\infty} A_{n} f_{n}(x) t^{n} \\
& =\frac{1}{2 \pi i} \int_{c} \frac{F(z)}{z-x} \sum_{n=0}^{\infty}\left[\frac{(a z+b) t}{z-x}\right]^{n} \sum_{k=0}^{n n / N]}\left(\begin{array}{c}
n \\
N k
\end{array}\right) \lambda_{k} y^{k} d z \\
& =\frac{1}{2 \pi i} \int_{C} \frac{F(z)}{z-x} \sum_{k=0}^{\infty} \lambda_{k} y^{k}\left[\frac{(a z+b) t}{z-x}\right]^{N k} \sum_{n=0}^{\infty}\left(\begin{array}{c}
n+N k \\
N k
\end{array}\right)\left[\frac{(a z+b) t}{z-x}\right]^{n} d z \\
& =(1-a t)^{-1} \sum_{k=0}^{\infty}\left[\frac{y t^{N}}{(1-a t)^{N}}\right]^{k} \frac{1}{2 \pi i} \int_{C} \frac{(a z+b)^{N k} F(z) d z}{\{z-(x+b t) /(1-a t)\}^{N k+1}},
\end{aligned}
$$

by the familiar binomial expansion, and the generating-function equivalence (10) follows, since the pole at $z=(x+b t) /(1-a t)$ can always be placed inside $C$ by taking $t$ sufficiently small and then the result extended by analytic continuation on $t$.

We remark in passing that Theorem 2 will yield the generating-function equivalences contained in Theorem 1 when the arbitrary coefficients $\lambda_{n}$ are specialized by

$$
\lambda_{n}=\frac{\left(\alpha_{1}\right)_{n} \ldots\left(\alpha_{p}\right)_{n}}{\left(\beta_{1}\right)_{n} \ldots\left(\beta_{q}\right)_{n}}, \quad n=0,1,2, \ldots,
$$

$y$ is replaced by $-y$, and the arbitrary positive integer $N$ is set equal to 1 or 2 . 
2. Applications. Since a fairly large number of special functions satisfy a Rodrigues formula of type (1), the generating-function equivalences given by Theorem 2 are widely applicable. We content ourselves by noting the familiar instances

$$
\begin{aligned}
f_{n}(x) & =\frac{(-1)^{n} e^{-x^{2}}}{n !} H_{n}(x)=\frac{1}{n !} D_{x}^{n}\left\{e^{-x^{2}}\right\} \\
{ }_{n}(x) & =x^{\alpha} e^{-x} L_{n}^{(\alpha)}(x)=\frac{1}{n !} D_{x}^{n}\left\{x^{n+\alpha} e^{-x}\right\}, \\
f_{n}(x) & =x^{\alpha-n} e^{-x} L_{n}^{(\alpha-n)}(x)=\frac{1}{n !} D_{x}^{n}\left\{x^{\alpha} e^{-x}\right\} \\
f_{n}(x) & =2^{n}(x-1)^{\alpha}(x+1)^{\beta-n} P_{n}^{(\alpha, \beta-n)}(x) \\
& =\frac{1}{n !} D_{x}^{n}\left\{(x-1)^{n+\alpha}(x+1)^{\beta}\right\}, \\
f_{n}(x) & =2^{n}(x-1)^{\alpha-n}(x+1)^{\beta} P_{n}^{(\alpha-n, \beta)}(x) \\
& =\frac{1}{n !} D_{x}^{n}\left\{(x+1)^{n+\beta}(x-1)^{\alpha}\right\}, \\
f_{n}(x) & =2^{n}(x-1)^{\alpha-n}(x+1)^{\beta-n} P_{n}^{(\alpha-n, \beta-n)}(x) \\
& =\frac{1}{n !} D_{x}^{n}\left\{(x-1)^{\alpha}(x+1)^{\beta}\right\},
\end{aligned}
$$

involving the classical orthogonal polynomials of Hermite, Laguerre, and Jacobi. The results of the preceding section would apply also to the ultraspherical polynomials $P_{n}^{\alpha}(x)$, the Legendre polynomials $P_{n}(x)$, and the Bessel polynomials $y_{n}(x, \alpha-n, \beta)$, since we have

$$
\begin{gathered}
f_{n}(x)=(-1)^{n}\left(x^{2}-1\right)^{-\alpha-n / 2} P_{n}^{\alpha}\left(x / \sqrt{ }\left(x^{2}-1\right)\right) \\
=\frac{1}{n !} D_{x}^{n}\left\{\left(x^{2}-1\right)^{-\alpha}\right\}, \\
P_{n}(x)=P_{n}^{ \pm}(x),
\end{gathered}
$$

and (cf.[2], p. 111, Equation (47))

$$
\begin{aligned}
f_{n}(x) & =\frac{\beta^{n} x^{\alpha-n-2} e^{-\beta / x}}{n !} y_{n}(x, \alpha-n, \beta) \\
& =\frac{1}{n !} D_{x}^{n}\left\{x^{n+\alpha-2} e^{-\beta / x}\right\} .
\end{aligned}
$$


Thus, in each of the aforementioned cases, one can easily apply the lemma as well as Theorem 2 to derive a (known) generating relation of type (6) and a new class of generating-function equivalences of type (10). We omit the details.

On the other hand, for the sequence of hypergeometric functions

$$
g_{n}^{(\alpha, \beta)}(x)=\frac{(\alpha)_{n}}{n !}{ }_{2} F_{1}\left[\frac{1}{2} n+\frac{1}{2} \alpha, \frac{1}{2} n+\frac{1}{2} \alpha+\frac{1}{2} ; \beta ; x\right], \quad n=0,1,2, \ldots
$$

it is easily verified that

$$
\sum_{n=0}^{\infty}\left(\begin{array}{c}
m+n \\
n
\end{array}\right) g_{m+n}^{(\alpha, \beta)}(x) t^{n}=(1-t)^{-\alpha-m} g_{m}^{(\alpha, \beta)}\left(\frac{x}{(1-t)^{2}}\right), \quad m=0,1,2, \ldots,
$$

which is of type (6). Thus, as an analogue of the generating-function equivalence (10), we have

$$
\sum_{n=0}^{\infty} A_{n} g_{n}^{(\alpha, \beta)}(x) t^{n}=(1-t)^{-\alpha} \sum_{n=0}^{\infty} \lambda_{n} g_{N n}^{(\alpha, \beta)}\left(x /(1-t)^{2}\right)\left[y t^{N} /(1-t)^{N}\right]^{n},
$$

where the $A_{n}$ are given by (8).

In view of the familiar Gaussian transformation

$$
{ }_{2} F_{1}[\alpha, \beta ; \gamma ; z]=(1-z)^{\gamma-\alpha-\beta}{ }_{2} F_{1}[\gamma-\alpha, \gamma-\beta ; \gamma ; z], \quad|z|<1,
$$

it follows from (22) that

$$
g_{n}^{(2 \alpha+k, \alpha+1 / 2)}\left(\left(x^{2}-1\right) / x^{2}\right)=\frac{(n+k) ! x^{n+k+2 \alpha}}{n !(2 \alpha)_{k}} P_{n+k}^{\alpha}(x), \quad n, k=0,1,2, \ldots
$$

since $P_{n}^{\alpha}(x)$ may be defined by

$$
P_{n}^{\alpha}(x)=\frac{(2 \alpha)_{n} x^{n}}{n !}{ }_{2} F_{1}\left[-\frac{1}{2} n,-\frac{1}{2} n+\frac{1}{2} ; \alpha+\frac{1}{2} ;\left(x^{2}-1\right) / x^{2}\right] .
$$

Now the $A_{n}$ given by (8) can evidently be reduced in terms of the ultraspherical polynomials $P_{n}^{\beta}(x)$ if in (8) we set $N=2, \lambda_{n}=\left(\frac{1}{2}\right)_{n} /\left(\beta+\frac{1}{2}\right)_{n}$, and replace $y$ by $\left(y^{2}-1\right) / y^{2}$. Hence, by interpreting the first member of (24) with the help of (26), and the second member by means of (22), we shall arrive at the bilinear generating relation

$$
\begin{aligned}
\sum_{n=0}^{\infty} \frac{(n+k) !}{(2 \beta)_{n}} P_{n+k}^{\alpha}(x) P_{n}^{\beta}(y) t^{n}=(2 \alpha)_{k}(x-y t)^{-2 \alpha-k} \\
\quad \cdot F_{4}\left[\alpha+\frac{1}{2} k, \alpha+\frac{1}{2} k+\frac{1}{2} ; \alpha+\frac{1}{2}, \beta+\frac{1}{2} ; \frac{x^{2}-1}{(x-y t)^{2}}, \frac{\left(y^{2}-1\right) t^{2}}{(x-y t)^{2}}\right],
\end{aligned}
$$

where $k=0,1,2, \ldots$, and $F_{4}$ denotes the fourth type of Appell's functions defined by

$$
F_{4}\left[\alpha, \beta ; \gamma, \gamma^{\prime} ; x, y\right]=\sum_{m, n=0}^{\infty} \frac{(\alpha)_{m+n}(\beta)_{m+n}}{(\gamma)_{m}\left(\gamma^{\prime}\right)_{n}} \frac{x^{m}}{m !} \frac{y^{n}}{n !} .
$$




\section{REFERENCES}

1. F. Brafman, Generating functions and associated Legendre polynomials, Quart. J. Math. (Oxford) (2) 10 (1959), 156-160.

2. H. L. Krall and O. Frink, A new class of orthogonal polynomials: The Bessel polynomials, Trans. Amer. Math. Soc. 65 (1949), 100-115.

3. E. T. Whittaker and G. N. Watson, $A$ Course of Modern Analysis, Fourth edition (Cambridge, 1963).

Department of Mathematics

UNIVERSITY OF VICTORIA

Victoria, British Columbia, Canada

V8W 2 Y2 